\title{
Comparison of Properties of Single Jersey Knit Fabrics Knitted With Single and Double Ply Yarn of Same Resultant Count
}

\author{
Mohammad Hosain Reza, \\ Assistant Professor, Department of Textile Engineering, \\ Daffodil International University. Dhaka, Bangladesh.
}

doi: 10.19044/esj.2017.v13n18p473 URL:http://dx.doi.org/10.19044/esj.2017.v13n18p473

\begin{abstract}
The constructional properties of a weft knitted fabric depends on stitch length and yarn count. In this paper it is searched that whether yarn ply affects the properties of weft knitted fabric though knitted from yarns of same resultant count. For this fabric samples were knitted with pairs of single ply and double ply yarns with the same resultant count. Then the constructional properties like course / cm, wales / cm, stitch density, G.S.M. and spirality were measured. 10 data for each property from each sample were taken and averaged. From the averaged data graphs were constructed to make the comparison. From the graphs it is found that the properties (i.e. is course / cm, wales / cm, stitch density, G.S.M) is less in all of the sample knitted from double ply yarns than the fabric samples knitted from single ply yarns. Moreover though the fabric samples were made by the same settings of knitting machine but the stitch length is more in fabric samples knitted by double ply yarns. As the component yarns lose twist during doubling and twisting they become bulkier. For this stitches occupied more spaces in fabric made from double ply yarn. This lead to make the difference in constructional properties between fabric samples made from single ply yarns and double ply yarns of same resultant count.
\end{abstract}

Keywords: Single ply yarn, Double ply yarn, Course / cm, Wales / cm, Stitch density, Stitch length, G.S.M., Spirality, Resultant yarn count, Constructional properties

\section{Limitations}

The single ply yarns used in this study were rotor yarns while the double ply yarns were combed yarns. Moreover, appropriate machine gauge could not be used for different yarn counts during knitting the samples. 


\section{Introduction}

The constructional properties of a weft knitted fabric depend on yarn count and stitch length. If any of the parameter varies, the properties of knit fabric will vary. The purpose of this work was to compare the constructional properties of knit fabric produced by single ply yarn and double ply yarns with the same resultant count.

\section{Experimental Details}

\section{Yarn count}

Yarn count is a numerical expression, which express the coarseness or fineness that is whether the yarn is thick or thin. To find out the resultant count of a ply yarn the following formula is used in indirect systems like English cotton count:

$$
\frac{1}{N R}=\frac{1}{N 1}+\frac{1}{N 2}+\frac{1}{N 3}+\cdots \ldots \ldots \ldots+\frac{1}{N n}
$$

Where $\mathrm{N}_{\mathrm{R}}=$ Resultant yarn count (in indirect system)

And $\mathrm{N}_{1}, \mathrm{~N}_{2}, \mathrm{~N}_{3}=$ Yarn count of component single yarns. (in indirect system)

However, in direct count system like tex or denier the formula is as follows:

$\mathrm{N}_{\mathrm{R}}=\mathrm{N}_{1}+\mathrm{N}_{2}+\mathrm{N}_{3}+\ldots \ldots \ldots \ldots+\mathrm{N}_{\mathrm{n}}$

Where $\mathrm{N}_{\mathrm{R}}=$ Resultant Yarn count (Direct System of yarn count)

And $\mathrm{N}_{1}, \mathrm{~N}_{2}, \mathrm{~N}_{3}=$ Yarn count (in direct system) of component single yarns.

\section{Course per cm}

A course is a predominantly horizontal row of loops produced by the adjacent needles during the same knitting cycle. In a brief, courses are rows of loops across the length of the fabric. It is measured along the wales direction by needle and counting glass. 10 readings were taken for course per $\mathrm{cm}$ from each sample. They were then averaged to get the final data.

\section{Wales per cm}

A Wale is a vertical column of intermeshed needle loops produced by the same needles knitting at successive knitting cycle. In a brief, wales are columns of loops across the width of the fabric. It is measured along the course direction by needle and counting glass. 10 readings were taken for wales per $\mathrm{cm}$ from each sample. They were then averaged to get the final data. 


\section{Stitch density}

Stitch density means the total number of loops in a measured area of fabric. (Square inch or square centimeter). It can be also obtained by counting number of courses per $\mathrm{cm}$ and number of wales per $\mathrm{cm}$, then multiplying them. By the help of a counting glass 10 readings were taken for stitch density from each sample. They were then averaged to get the final data.

\section{Stitch length}

Stitch length is the length of yarn knitted into a complete stitch. A stitch is comprises of a needle loop and half of two sinker loops. To determine the stitch length the following procedure was followed:

a) 20 loops were counted at the edge of the fabric, across the course direction.

b) Start and the end point of the 20 loops were marked on yarn which is bent into loops.

c) The loops were then unraveled and straitened to yarn.

d) The distance between the start and end marks on the yarn were measured in mm.

e) Finally, the lengths measured in mm, were divided by 20 (number of loops) to obtain the stitch length. In this way 10 readings were taken for stitch length from each sample. They were then averaged to get the final data.

\section{G.S.M}

The property is self-explanatory which indicates the weight in grams, of one square meter fabric. To determine the G.S.M. of the fabric samples the following procedure was followed:

a) A rectangular piece of fabric sample was cut from the main sample.

b) Its length and width were measured and recorded in nearest $\mathrm{mm}$.

c) The sample was then weighted by the help of a digital balance to nearest gm.

d) Finally the G.S.M. was calculated with the help of following formula:

$$
\text { G.S.M. }=\frac{\text { Sample weight in } \mathrm{gm}}{\text { Length } \times \text { width in } \mathrm{mm}} \times 1000 \times 1000
$$

\section{Spirality}

In a knitted fabric there are many loops in it. The horizontal rows of loops are called "A Course". And the vertical columns of loops are called "A Wale". According to the definition the "A wale" and "A course" of a knitted fabric should lie perpendicular to each other. But due to some causes wale 
line doesn't always stand perpendicular to the course. This phenomenon is called spirality.

In other words, spirality occurs in knitted fabric because of asymmetric loops which turns in the wales and course of a fabric into an angular relationship other than 90 degree. The angle between wale and a line drawn perpendicular to the course is the measure of spirality.

To measure the spirality a protractor was used to find the angles in degree between the wales and the line drawn perpendicular to the courses.

At the beginning $3 \mathrm{knit}$ fabric samples were made from single ply yarns with yarn count (Ne) $10 / 1,16 / 1,20 / 1$. Then in the same way another 3 fabric samples were made from double ply yarns with yarn count (Ne) $20 / 2,32 / 2$ and $40 / 2$. It is mention-able that $10 / 1$ (Ne) and $20 / 2$ (Ne) have the same resultant yarn count, which is $10(\mathrm{Ne})$. Similarly, $16 / 1$ and 32 / 2 have the same resultant yarn count, which is $16(\mathrm{Ne})$. Again $20 / 1$ and 40 / 2 have the same resultant yarn count, which is $20(\mathrm{Ne})$.

A hose knitting machine of 3.75 inch diameter and 6 gauge was used to produce all the fabric samples. During producing the samples same settings were maintained in the machine.

After producing the fabric samples, they were allowed to relax for 24 hours in normal atmospheric conditions. Then the constructional properties course $/ \mathrm{cm}$, wales $/ \mathrm{cm}$, stitch density, G.S.M. and spirality were measured and recorded from each samples. At the same time stitch length of all of the samples were measured and recorded. For each specification and properties 10 data were collected and averaged. They were then compared and graphically represented according to corresponding samples produced from same resultant yarn counts.

The data taken from the samples were averaged. The averaged data were plotted in graphs. From the graphs the individual constructional properties of knit fabrics of single ply yarns and double ply yarns were compared.

\begin{tabular}{|c|c|c|c|c|c|c|}
\hline & \multicolumn{6}{|c|}{ Resultant Yarn Count (Ne) } \\
\hline & \multicolumn{2}{|c|}{10} & \multicolumn{2}{|c|}{16} & \multicolumn{2}{|c|}{20} \\
\hline & $\begin{array}{c}\text { Single } \\
\text { ply } \\
\text { Yarn, } \\
\mathrm{Ne}= \\
10 / 1\end{array}$ & $\begin{array}{c}\text { Double } \\
\text { ply } \\
\text { Yarn, } \\
\mathrm{Ne}= \\
20 / 2\end{array}$ & $\begin{array}{c}\text { Single } \\
\text { ply } \\
\text { Yarn, } \\
\mathrm{Ne}= \\
16 / 1\end{array}$ & 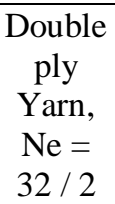 & $\begin{array}{l}\text { Single } \\
\text { ply } \\
\text { Yarn, } \\
\mathrm{Ne}= \\
20 / 1\end{array}$ & 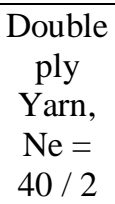 \\
\hline Course / cm & 6.23 & 6.2 & 6.3 & 6.1 & 6.2 & 6 \\
\hline Wales / cm & 6.4 & 5 & 7.2 & 5.5 & 7.3 & 6 \\
\hline Stitch density (per Square cm) & 35.8 & 30.99 & 38.28 & 33.24 & 38.95 & 32.36 \\
\hline Stitch length (mm) & 7.16 & 7.33 & 7.3 & 7.33 & 7 & 7.69 \\
\hline
\end{tabular}




\begin{tabular}{|c|c|c|c|c|c|c|}
\hline Gram per square meter & 178 & 126 & 124 & 84 & 100 & 61 \\
\hline Spirality (Degree) & 39.2 & 3 & 46.8 & 3 & 41.9 & 3 \\
\hline
\end{tabular}

Table 1: Constructional properties knit fabric samples produced from single ply yarns and double ply yarns with same resultant count $(\mathrm{Ne})$.

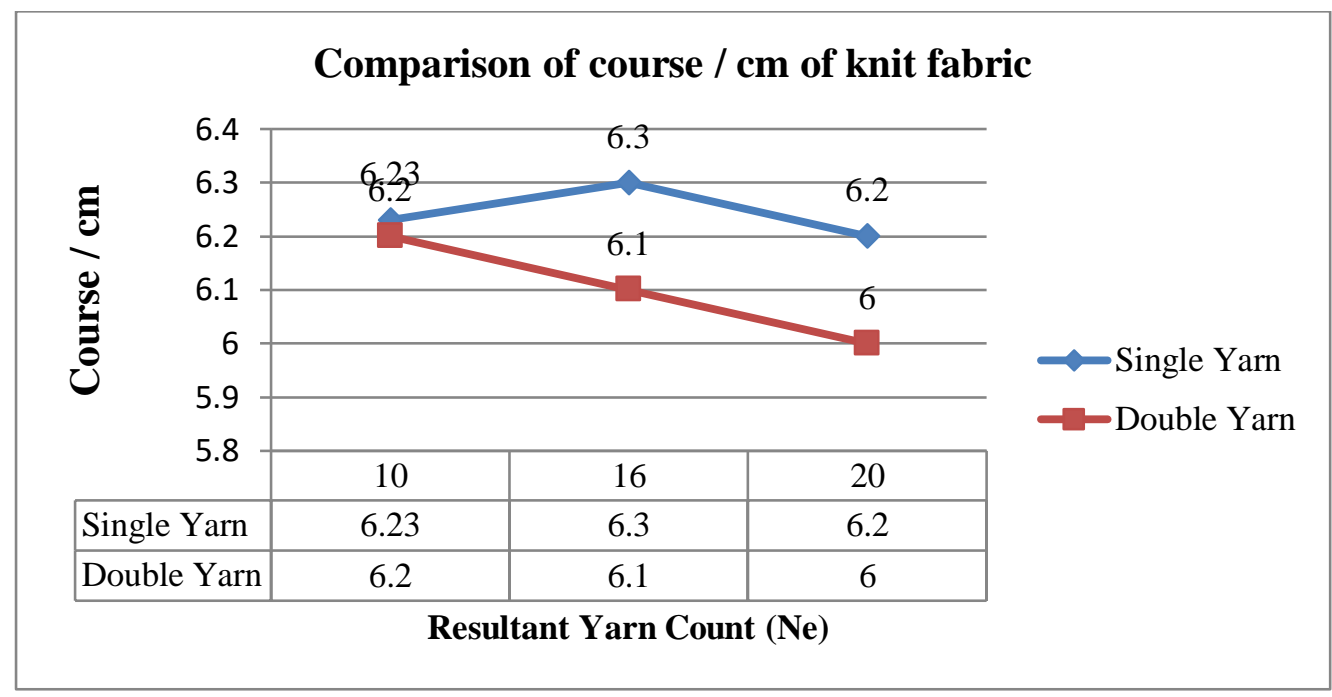

Figure 1: Comparison of course / $\mathrm{cm}$ of knit fabric sample produced from single ply yarn and double ply yarn with same resultant count.

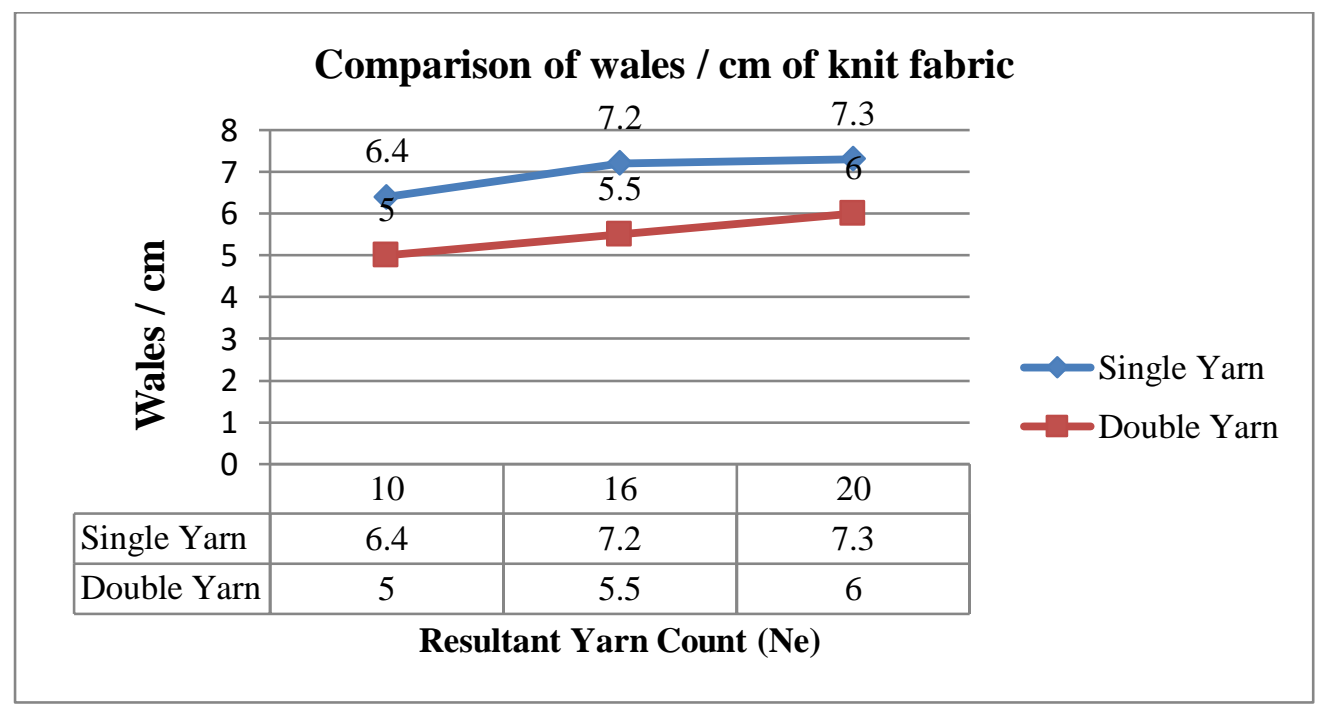

Figure 2: Comparison of wales / $\mathrm{cm}$ of knit fabric sample produced from single ply yarn and double ply yarn with same resultant count. 


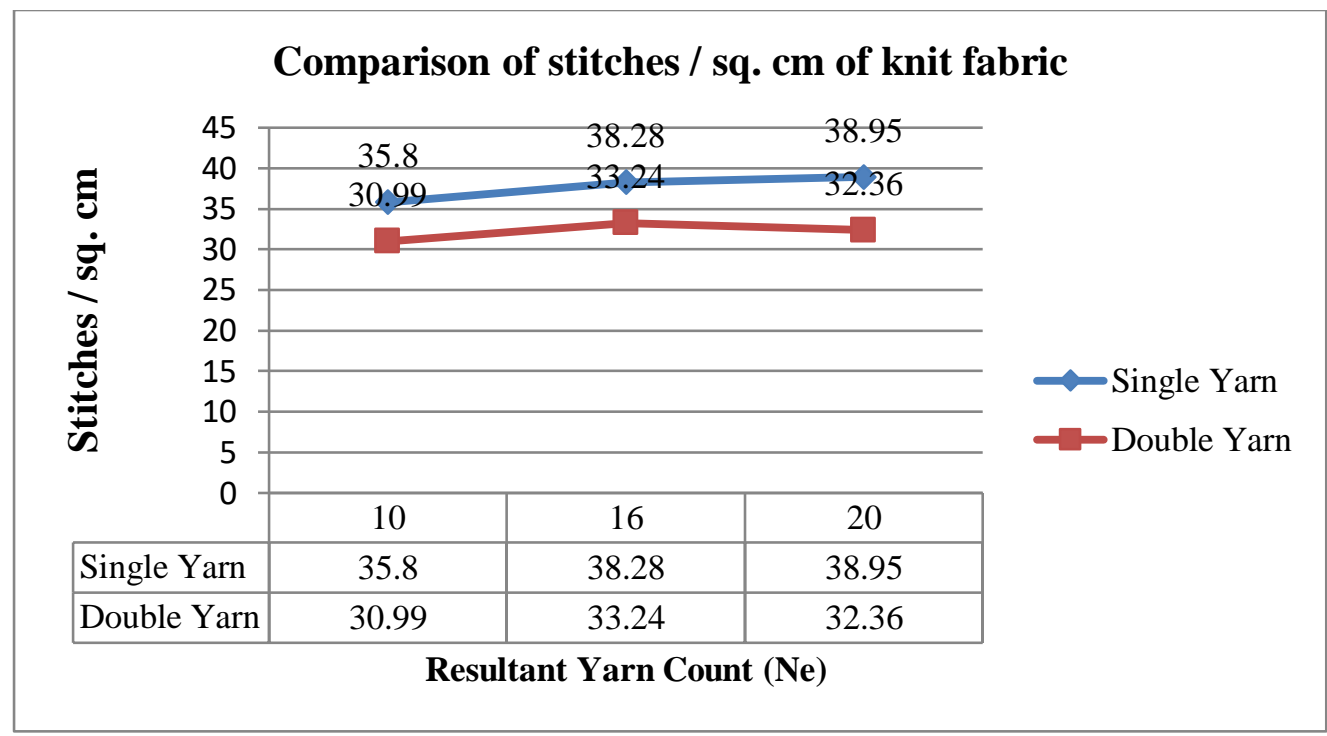

Figure 3: Comparison of stitch density per square $\mathrm{cm}$ of knit fabric sample produced from single ply yarn and double ply yarn with same resultant count.

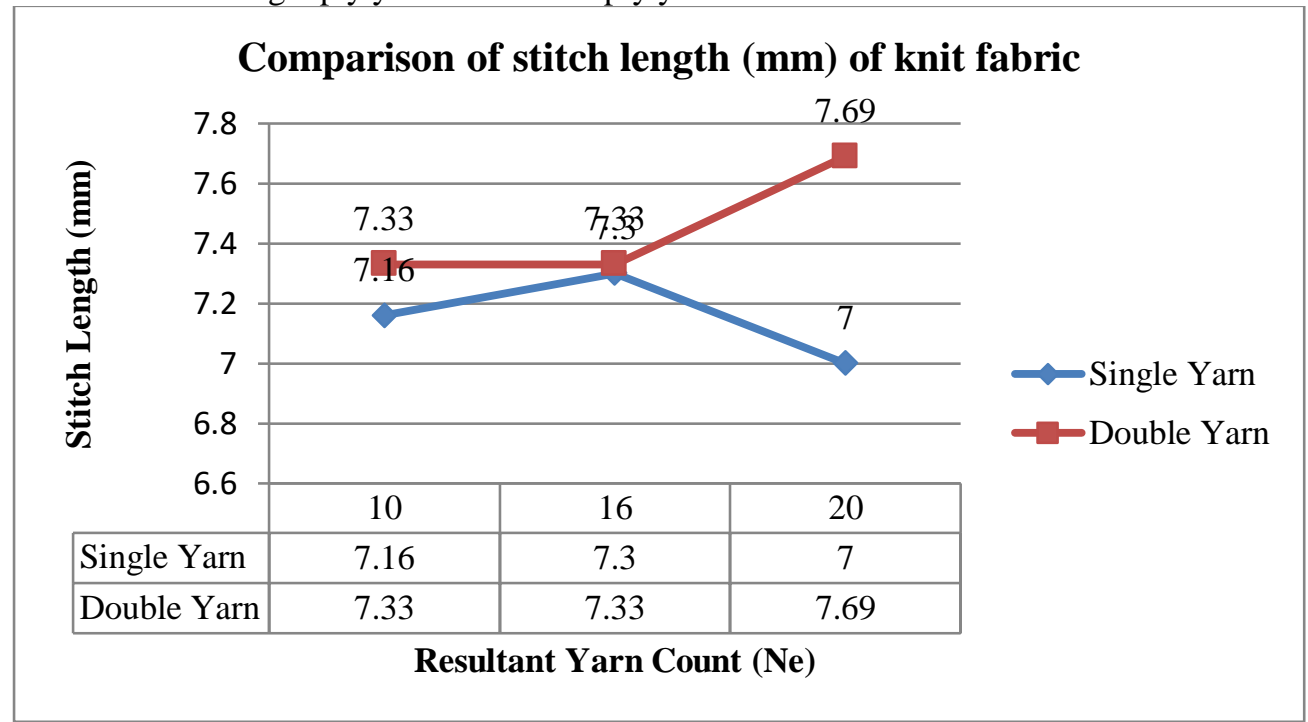

Figure 4: Comparison of stitch length $(\mathrm{mm})$ of knit fabric sample produced from single ply yarn and double ply yarn with same resultant count. 


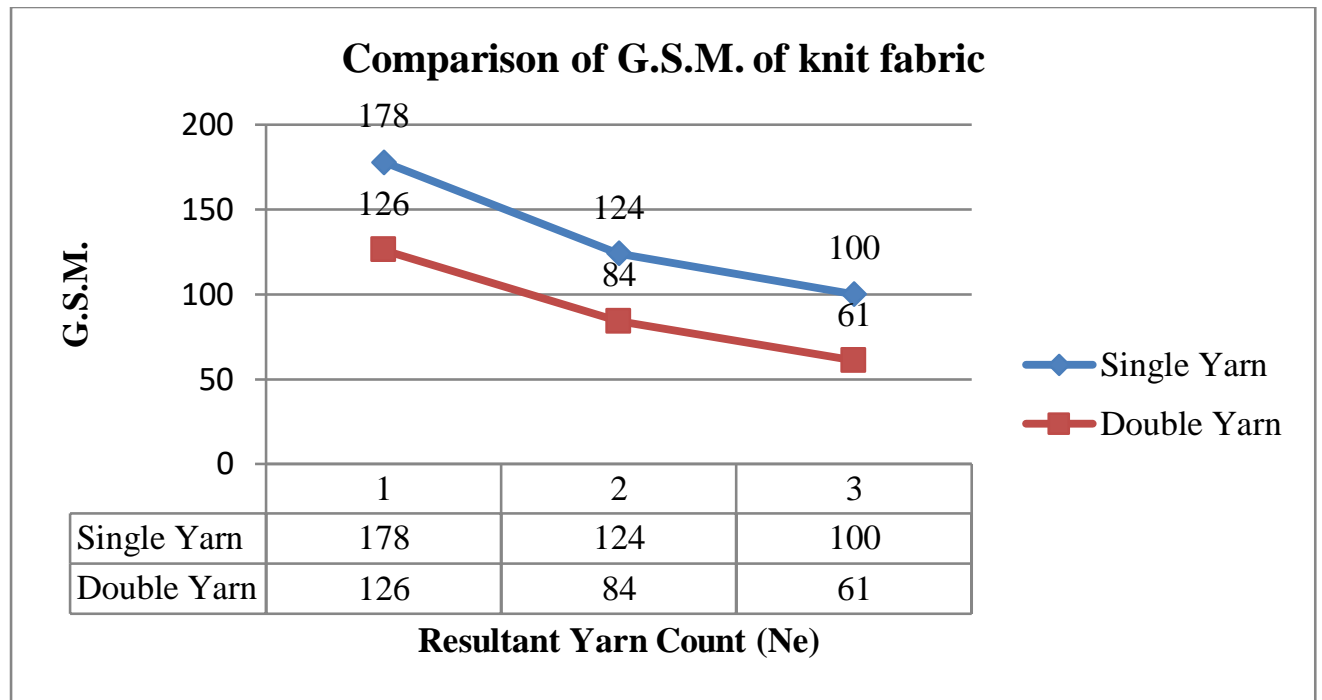

Figure 5: Comparison of G.S.M. of knit fabric sample produced from single ply yarn and double ply yarn with same resultant count.

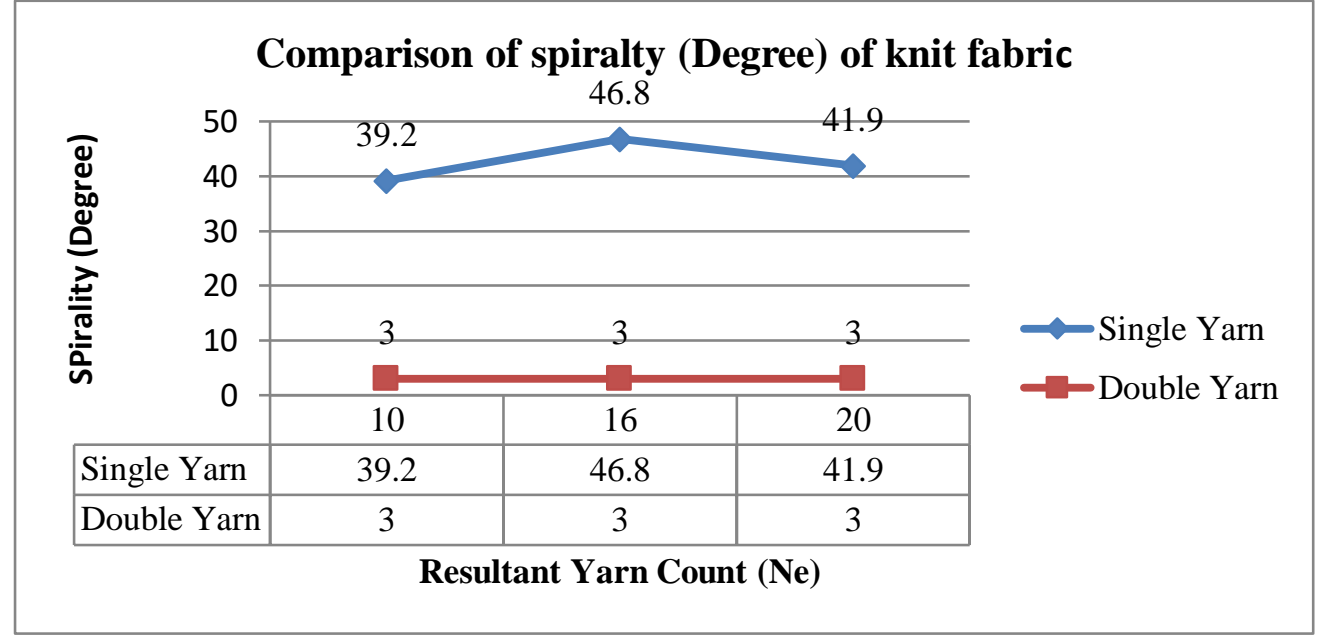

Figure 6: Comparison of spirality (Degree) of knit fabric sample produced from single ply yarn and double ply yarn with same resultant count.

\section{Result and discussion}

From the tables and graphs it is seen that constructional properties; i.e.; course $/ \mathrm{cm}$, wales $/ \mathrm{cm}$, stitches per square $\mathrm{cm}$, G.S.M. and spirality have higher values in knit fabric samples produced from single ply yarns than the samples produced from double ply yarns with corresponding same resultant count.

The constructional properties of any weft knitted fabric depend on the stitch length and yarn count.

From Table 1 and Figure 4 it is seen that the stitch length is less in knit fabric samples produced from single ply yarns than their corresponding 
samples produced from double ply yarns with same resultant count. As the stitch length is less, so more loops can be accommodated in unit length of fabric. The stitch length is less in samples produced from single ply yarns results in higher values of course / $\mathrm{cm}$ and wales $/ \mathrm{cm}$ in the samples produced from single ply yarns. For the same reason stated above, the stitch density per square $\mathrm{cm}$ is also more in samples produced from single ply yarns than the samples produced from double ply yarns.

Again as the stitch length is inversely proportional to G.S.M., the G.S.M. is more (as stitch length is less) in samples produced from single ply yarns than the samples produced from corresponding double ply yarns. It is mention-able that the both groups of sample were made from yarns of same resultant count.

The reason behind the less stitch length in samples produced from single ply yarns is because the yarns are more compact in case of single ply yarns than their corresponding double ply yarns. During doubling and twisting the double ply yarns, opposite twist of component yarn is inserted in the resultant double ply yarn. The component single yarns are composed of ' $\mathrm{S}$ ' twist while composing the double ply yarn, ' $\mathrm{Z}$ ' twist is inserted. As a result the component single yarns lose their twist and become bulkier. For this reason, though the double ply yarns have same resultant count as the single ply yarns, the double ply yarns becomes bulkier than corresponding single ply yarns. This is why stitch length of double ply yarns samples occupies more spaces in fabric than the stitch length of single ply yarns.

This result in more course / cm, wales / cm, stitch density and G.S.M. in samples produced from single ply yarns.

Again the main reason behind spirality in knit fabric is yarn twist. As the component single yarns in double yarns lose twist during doubling and twisting the spirality becomes less in the samples produced from double ply yarns. Moreover, the ' $Z$ ' twist inserted during doubling and twisting neutralizes the effects of ' $\mathrm{S}$ ' twist in component single yarns. This results in less or nearly no spirality in samples produced by double ply yarns. Whereas the samples produced from single ply yarns exhibits a great extent of spirality.

\section{Conclusion}

As the component yarns lose twist during composing the double ply yarns by doubling and twisting, it becomes bulkier. So, the stitch length occupies more spaces in samples produced from double ply yarns than their corresponding single ply yarn fabric samples. In this work it is found that the course / cm, wales / cm, stitch density, G.S.M and spirality is less in samples produced from double ply yarns than the corresponding samples of single 
yarns of same resultant count. Again spirality is almost nil in the samples produced from double ply yarns.

\section{References:}

1. Spencer, David J., "Knitting Technology", Third Edition, 2001, WOODHEAD PUBLISHING LIMITED, Cambridge, England.

2. Iyer, Mammel, and Schach, "Circular Knitting", Second Edition, 2004, Meisenbach GmbH, Bamberg, Germany.

3. Ambumani N., "Knitting Fundamentals, Machines, Structures and Developments", First Edition, 2007, NEW AGE INTERNATIONAL PUBLISHERS, New Delhi, India.

4. Booth, J.E., "Principles of Textile Testing", Third Edition, NEWNES_BUTTERWORTHS, London. 